\title{
Ethik der Unternehmensführung
}

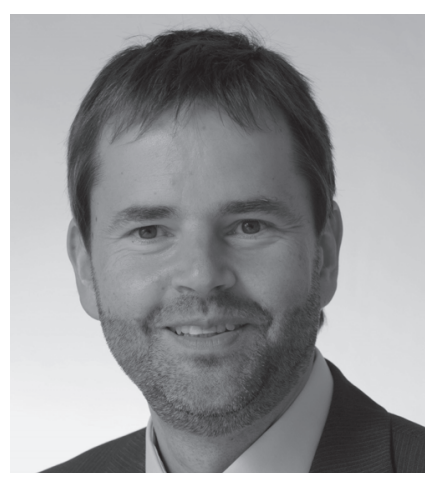

VON ROBERT BACHERT UND LUISA SANDRITTER

Robert Bachert ist Finanzvorstand des Diakonischen Werkes Württemberg. Davor war er in gleicher Funktion beim Diakonischen Werk Baden tätig.

www.diakonie-wuerttemberg.de

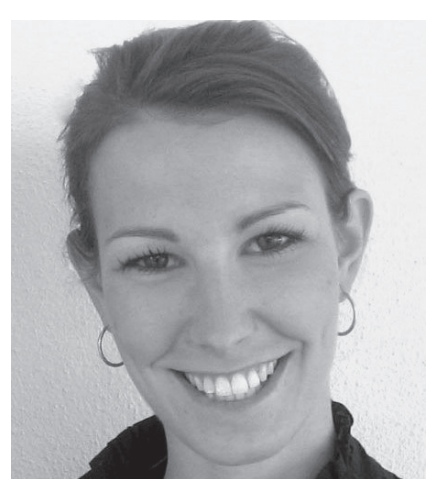

Luisa Sandritter ist Referentin Krisenberatung im Diakonischen Werk Baden.

www.diakonie-baden.de

\author{
Vor drei Jahren hat das Diakonische Werk Baden \\ einen Corporate Governance Kodex verabschiedet. \\ Zwei Umfragen zeigen, dass die entsprechenden \\ Empfehlungen bei den Mitgliedsorganisationen \\ weitgehend umgesetzt worden sind. Der Kodex \\ hat sich in einigen Fällen als Auslöser für eine \\ erfolgreiche Organisationsentwicklung erwiesen.
}

Die Vermeidung von Unternehmenskrisen in Wirtschaft, Industrie und Sozialbranche erfordern die Einhaltung von Standards guter Unternehmensleitung und Unternehmensaufsicht. Für die Diakonie in Baden wurden diese Anfang 2009 in einem Corporate Governance Kodex verabschiedet. Der Frage wie sich die Umsetzung solcher Empfehlungen in der Realität von Mitgliedseinrichtungen vollzieht, gingen zwei Umfragen des Landesverbandes nach. Die Ergebnisse zeigen sehr gute Resultate in den Bereichen der Satzungs- und Geschäftsordnungsstruktur sowie Controlling und Risikomanagement, aber auch die Notwendigkeit einer ethischen und werteorientierten Weiterentwicklung.

\section{Ergebnisse}

Vor Einführung des Kodex der Diakonie Baden wurde festgestellt, dass etwa 90 Prozent der Einrichtungen zum Untersuchungszeitpunkt über ein Aufsichtsorgan verfügten. Hierunter wurden unter anderem ehrenamtliche Vorstände sowie Mitglieder- und Gesellschafterversammlung gefasst. Schriftliche Regelungen zur Zusammenarbeit zwischen Leitung und Aufsicht existierten in etwa zwei Drittel dieser Organisationen. Instrumente des Controllings waren mehrheitlich im Einsatz, in einzelnen Bereichen aber nicht optimal repräsentiert. Das Erkennen von und der Umgang mit Risiken wurden vornehmlich durch das Top-Management wahrgenommen.

Eine aktuelle Analyse sollte ermitteln, welche Empfehlungen des Regelwerks von den Mitgliedseinrichtungen verwirklicht werden und welche nicht. Man versprach sich aus den Antworten Hinweise auf einen inhaltlichen Veränderungs- und Ergänzungsbedarf sowie Impulse zur Weiterentwicklung des badischen Corporate Governance Kodexes.

Aus den Resultaten lassen sich überwiegend positive Rückschlüsse auf den Umgang mit Corporate Governance ziehen. Drei Viertel der Einrichtungen gaben an, den Kodex zu realisieren. Dabei wurde eine Umsetzung von 90 Prozent beim Leitungsgremium und von 80 Prozent beim Aufsichtsgremium für das jeweilige Tätigkeitsfeld erreicht.

Insbesondere im Bereich des Controllings zeigt sich im Vergleich zur Analyse 2008 eine deutliche Zunahme des Instrumenteneinsatzes. Dort erfuhren die Kostenstellenrechnung, die Wirtschaftsplanung und das Berichtswesen mit Umsetzungsquoten von 90 Prozent den stärksten Anstieg. Besonders positiv ist der Einsatz eines Risikomanagementsystems und innerhalb dessen die Implementierung von Frühwarnsystemen.

\section{Überlegungen für die Weiterarbeit}

Die praktische Anwendung des Kodexes zeigt, dass die ethischen Werte, die sich in diversen diakonischen Kodizes wiederfinden - beispielsweise Kompetenz, Transparenz, Kommunikationsorientierung und Verantwortung - dem Proprium der Diakonie nicht hinreichend gerecht werden. Es stellt sich die Frage, ob nicht ausdrücklich in der Diakonie weitere Werte, die das Proprium der Diakonie ausmachen, hinzugenommen werden müssten. 
Aus einer werteorientierten Gesamtbetrachtung gibt es drei Ansatzpunkte für die ethische Weiterqualifizierung des aktuellen Corporate Governance Kodexes für die Diakonie:

- Auf der Mikroebene steht die Verbindung der Ethik mit dem Menschen im Vordergrund, da nur er Verantwortung übernehmen kann. Auch ein Corporate Governance Kodex kann nur realisiert werden, wenn Manager und Aufsichtsräte ihr Verhalten ändern und an den Regelungen ausrichten. Hier wird die Frage aufgeworfen, ob ein Kodex nicht durch individualethische Bausteine, beispielsweise über eine freiwillige Selbstverpflichtung, eine Ergänzung erfahren müsste in Form eines Führungskräftekodexes (vgl. Brink/Tiberius 2011).

- Daneben erfordert eine Ethik der Governance vier Phasen, um ein Ethik-Management-System in einem Unternehmen einzuführen. In einer ersten Phase gilt es die Identität bestimmenden Werte des Kodexes zu ermitteln, indem jeder kollektive Akteur beschrieben wird. Hieraus entsteht eine Fülle an relevanten und interdependenten Werten, die verhaltensmaßgeblich und vor allem richtungsweisend in konfliktären Situationen sind. Ein Werteviereck bündelt die so entwickelten Werte und kommuniziert diese in sogenannten intra, inter und extra Teams. Phase drei implementiert sie über Instrumente (Compliance-, Werteprogramm und Ethikaudit System). Phase vier schließlich organisiert den Wertewandel. Der Corporate Governance Kodex könnte hier als ein mögliches Instrument dienen. Er würde die generierten Werte aufnehmen, auf das Management der »Führung und Aufsicht « hin konkretisieren und somit ein integrierter Bestandteil eines neuen Corporate Governance Kodexes

\section{Literatur}

Alexander Bring, Victor A. Tiberius: Ethisches Management. Grundlagen eines wert(e)orientierten Führungskräfte-Kodex. Haupt Verlag, Bern 2011. 550 Seiten. 62,- Euro. ISBN 978-3-25806857-2.

Josef Wieland: Die Tugend der Governance (Hg.): Verlag Metropolis, Marburg 2006. 181 Seiten. 22,80 Euro. ISBN 978-3-89518-546-5. der Diakonie werden (vgl. Wieland 2006).

- Und schließlich ist im Bund als Metaebene anzuregen, wie die Kontrolle der Einhaltung des Kodexes und die Ergreifung von Maßnahmen bei seiner Nichtanwendung aussehen und zukünftig realisiert werden können.

Die Diakonie Baden ist zuversichtlich, dass mit dem vorliegenden Kodex eine sehr gute Unternehmensführung gewährleistet ist und dass die durchgeführten und angedachten Maßnahmen zur verbesserten Anwendung im Satzungsbereich, im Controlling und im Risikomanagement führen. Die ethische Qualifizierung des Kodexes wird in einer zunehmend komplexeren Umwelt in den nächsten Jahren vordringliche Aufgabe des Gesamtvorstandes im Diakonischen Werk Baden sein.

\section{Gemeinsam in die Zukunft}

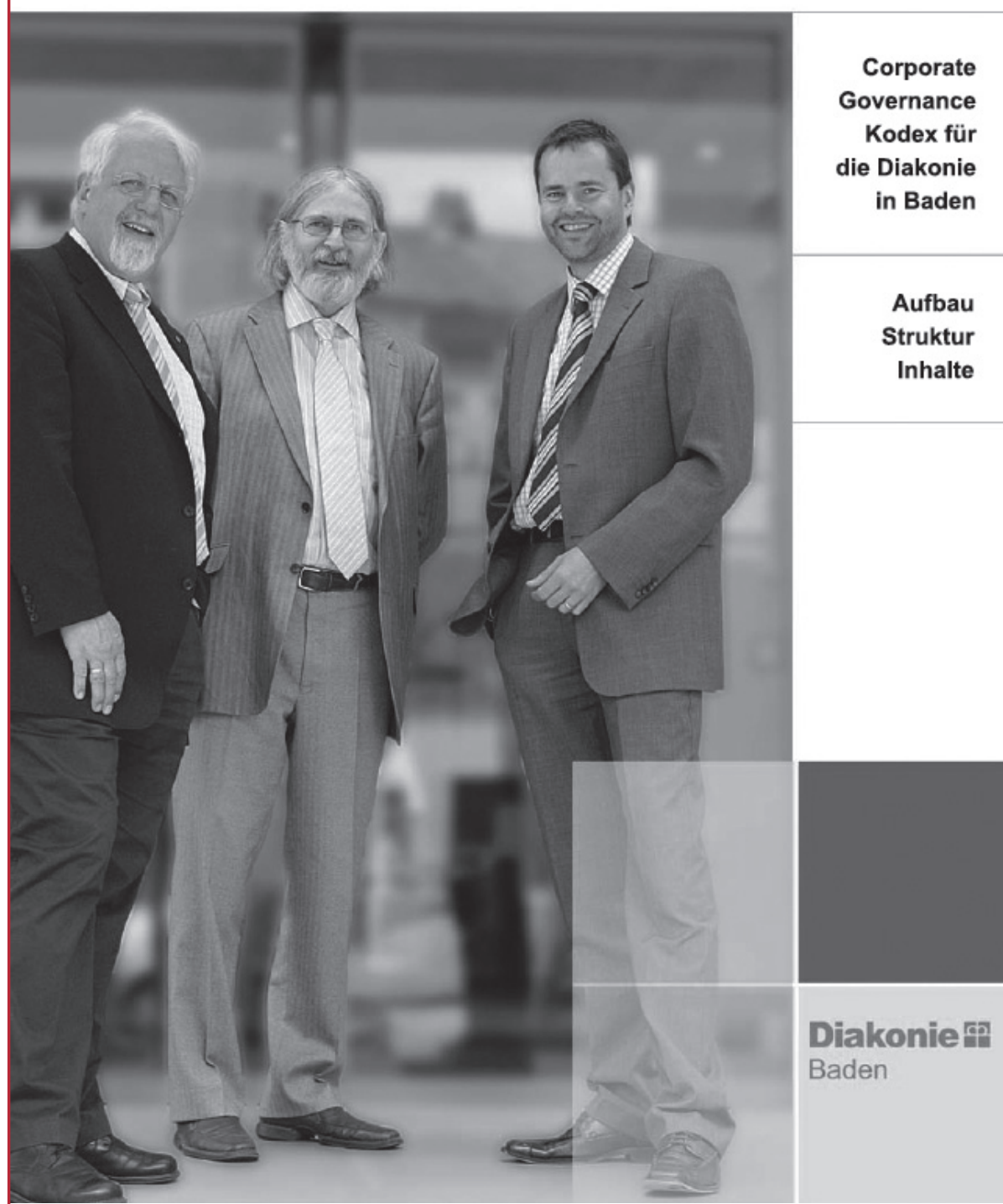

Der Corporate Governance Kodex für die Diakonie in Baden wurde vom Aufsichtsrat des Verbandes am 30. Januar 2009 verabschiedet. Mittlerweile haben sich die meisten Mitgliedsorganisationen mit den Grundsätzen guter Unternehmensführung auseinandergesetzt. Der Kodex steht als 15-seitiges PDF-Dokument auf der Website des Diakonischen Werkes Baden zum Herunterladen zur Verfügung.

www.diakonie-baden.de/fileadmin/documentpool/Diakonie_Baden/Corporate_Governance_Kodex-Diakonie_Baden.pdf 